\title{
The Measurement Challenge of the LHC Project
}

\author{
Gunnar Fernqvist
}

\begin{abstract}
In 2005, CERN is planning to commission its next accelerator project, the Large Hadron Collider. The project presents unprecedented challenges in the accurate control of currents up to $13 \mathrm{kA}$. Around 1700 superconducting magnet circuits are deployed in the $27 \mathrm{~km}$ circumference tunnel at CERN. A new concept of quasion-line calibration of the currents in the magnet circuits is proposed.
\end{abstract}

Index Terms-Accelerators, current control, current measurements.

\section{INTRODUCTION}

$\mathbf{C}$ ERN (The European Laboratory for Particle Physics) builds and operates particle accelerators for high-energy physics research. The particles are accelerated in circular orbits within a large ring-shaped vacuum enclosure. They are kept in their orbits by magnets (dipoles, quadrupoles etc.) powered by current-controlled power converters. The magnets are supplied with a programmed dc current waveform corresponding to the accelerating cycle. The accuracy, to which the currents in all the magnets are controlled, is fundamental to the successful accelerator operation and depends mainly on the measurement transducer, but also on the dynamic behavior of the power converters.

\section{THE LHC PROJECT}

The Large Hadron Collider (LHC) is a $7 \mathrm{TeV}$ proton-proton collider to be installed in the existing, $27 \mathrm{~km}$ circumference, accelerator tunnel by the year 2005 [1]. This particle energy demands the use of superconducting magnets producing a maximum field of $9 \mathrm{~T}$ at $13 \mathrm{kA}$ and cooled with superfluid helium at $1.8 \mathrm{~K}$. Practical limits in supplying liquid helium over long distances dictated the dividing of the machine into sectors. Due to the high electrical energy stored in the string of magnets (10 GJ in the dipoles), the magnet powering circuits also have to be divided into sectors to allow adequate protection. This leads to new difficulties in tracking of the magnet currents between the sectors.

The cost of the losses in the feeding cables would be prohibitive if the power converters were located in surface buildings. The converters will therefore be located underground, in galleries next to the accelerator tunnel.

\section{The LHC Powering ReQUiREMENT}

The LHC main magnets are split into eight equal sectors, with one dipole and two quadrupole circuits in each, resulting in 24 main circuits to be controlled and to track each other to the highest accuracy. The current cycle is depicted in Fig. 1. The most severe tolerance is required at the injection plateau

Manuscript received July 2, 1998.

The author is with CERN, Geneva 23, Switzerland.

Publisher Item Identifier S 0018-9456(99)03198-8. and the start of the ramp, which translates into an accuracy and stability of the order of $2 \times 10^{-6}$ of the maximum output current, $I_{\max }$. Further, due to the extreme sensitivity to beam losses at injection, the current control at the start of the ramp needs a resolution of $1 \times 10^{-6} I_{\max }$.

Once the finely adjusted current values have been found for best performance, they will be stored in computer files to repeat the accelerator cycle from hour to hour, day to day, month to month. The reproducibility of the currents is therefore important, as it will reduce setup time at every start-up of the accelerator. A summary of the estimated needs for the main magnets are as follows:

$$
\begin{array}{ll}
\text { Resolution: } & 1 \times 10^{-6} I_{\max }, \text { monotonic } \\
\text { Overshoot: } & \text { Zero }\left(<3 \times 10^{-6} I_{\max }\right) \\
\text { Stability, 30 min: } & \pm 2 \times 10^{-6} I_{\max } \\
\text { Reproducibility: } & \pm 5 \times 10^{-6} I_{\max } \\
\text { Tracking: } & \pm 5 \times 10^{-6} I_{\max } \\
\text { Accuracy: } & \pm 1 \times 10^{-5} I_{\max } .
\end{array}
$$

Further complications arise from static and dynamic errors in the magnet transfer function. Extensive magnetic measurements show that the field is not only dependent on the current, but also on its derivative and previous history. It is also likely that some systematic differences will be observed between magnets from different manufacturers. These will be quantified by magnetic measurements and used in correction algorithms. A central computer will calculate the vectors for control of all the power converters. Both the static and dynamic response of the converters need to be fully characterized.

\section{Present Power Converter Control}

Present methods of power converter control rely on many components, each being crucial to the performance. Fig. 2 shows a block-diagram. The control vectors from the central computer are downloaded to local function generators, that generate a string of digital control words in synchronism with the accelerator timing system. The digital control word is converted to an analog voltage in a DAC. The output current from the power converter is scaled down in a dc current transducer (DCCT) and then passed through a burden resistor. The resulting voltage is amplified and fed to the input of the control loop, where it is compared to the voltage from the DAC. The voltage is also fed to an ADC to monitor the output current. It would be very difficult to obtain the required accuracy of $1 \times 10^{-5} I_{\max }$ with this method.

\section{LHC POWER CONVERTER CONTROL}

Some of the necessary improvements can today be realized by using a sigma-delta ADC. This will provide the necessary 


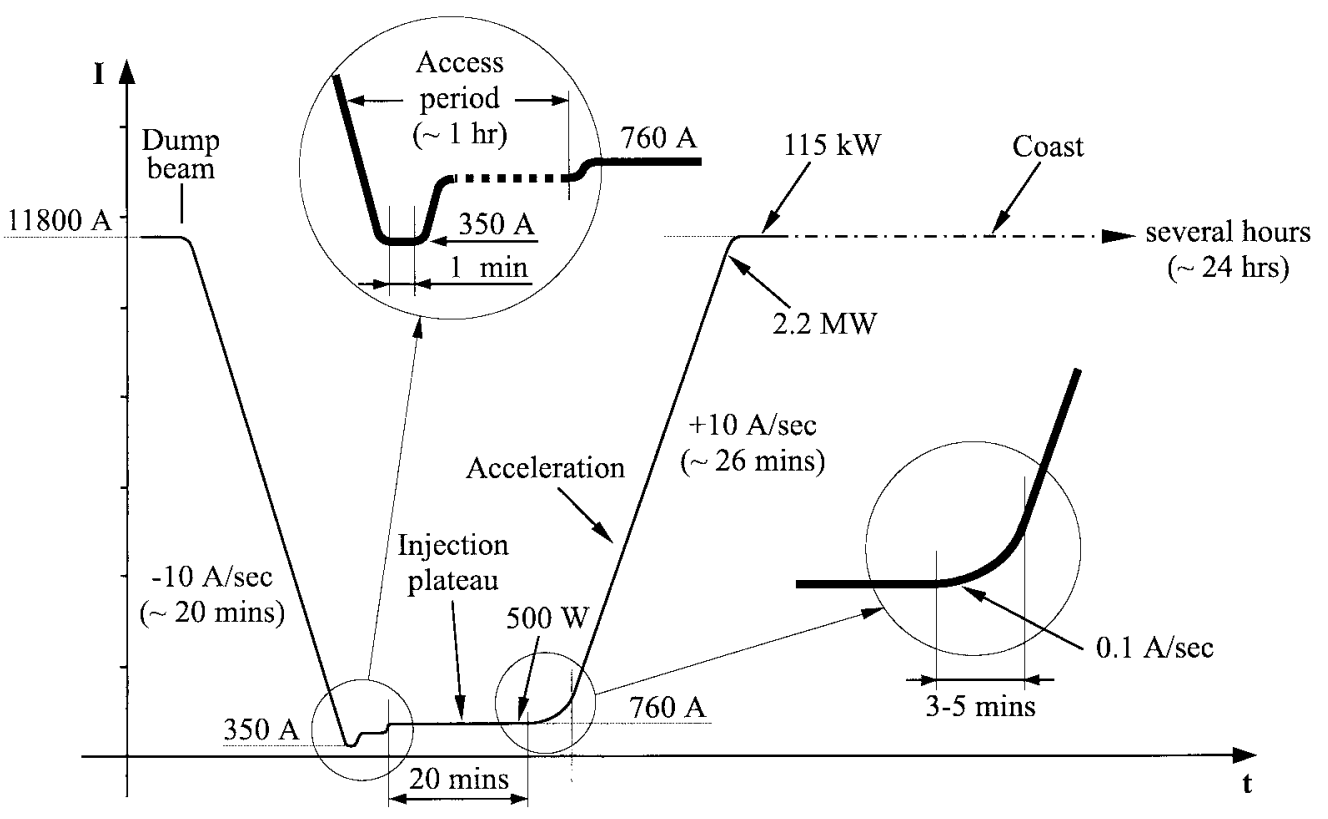

Fig. 1. The LHC dipole magnet current cycle.

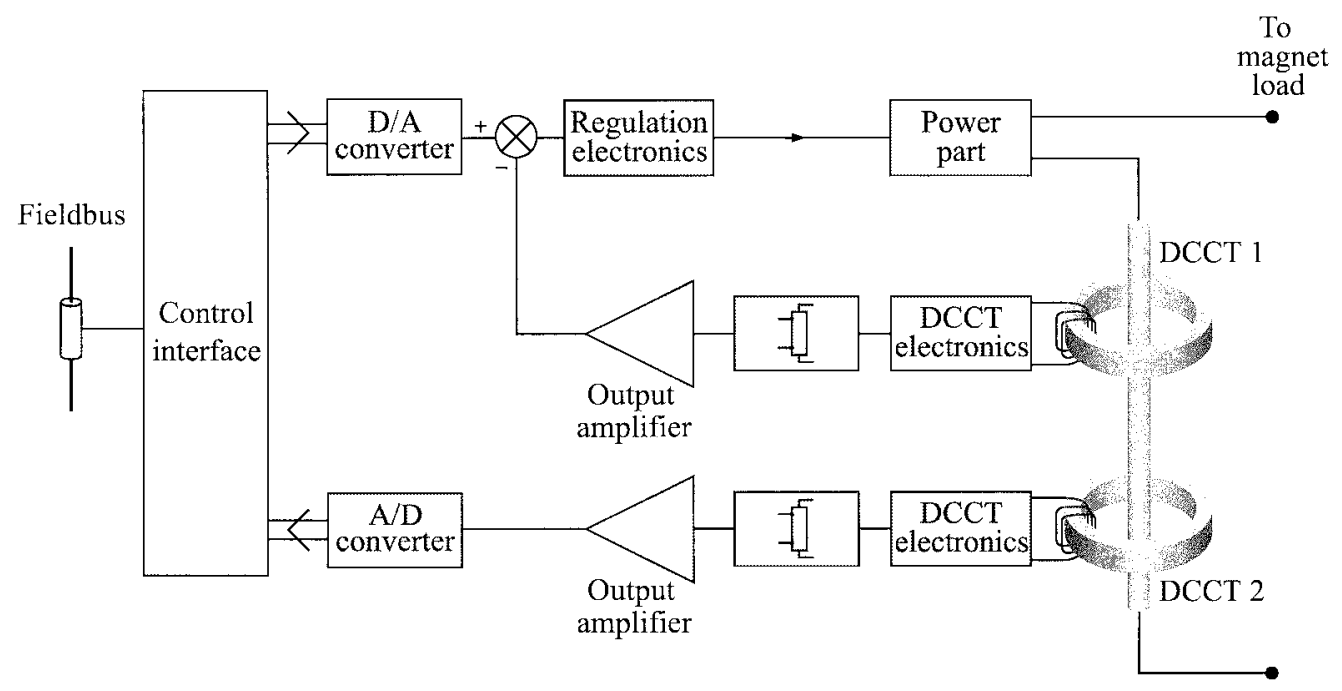

Fig. 2. Present power converter control.

resolution, monotonicity, linearity, and short-term stability. It is connected directly to the output of a specially designed DCCT providing the necessary performance. All the other previously critical parts of the control loop are realized in digital technology, eliminating any drift. Fig. 3 shows a blockdiagram.

The DCCT with its burden resistor and the ADC are then the only elements determining the performance. Two DCCT's and two ADC's will be used for redundancy. In a digital system it is easy to compare their outputs and choose either output for the regulation loop and/or the current monitor.

\section{CAlibration System}

A DCCT, like any high-precision transducer, needs frequent re-calibration, mainly due to zero drift and burden resistor aging, but also after repair or replacement. Removal of the DCCT becomes increasingly difficult as the current rating, and hence the size and weight, of the circuit increases. A new concept is proposed here, which will greatly simplify the quasion-line verification.

\section{A. DCCT Principle}

The DCCT works on the principle of flux compensation in a toroidal core encircling the current to be measured (see Fig. 4). For this purpose the core is equipped with a compensation winding fed from a feedback amplifier, whose input is a zeroflux detector. The ratio of the compensation current to the primary current is determined by the turns ratio to a relative uncertainty better than $1 \times 10^{-6}$, if the sensitivity of the zero-flux detector is sufficient. The compensation current is 


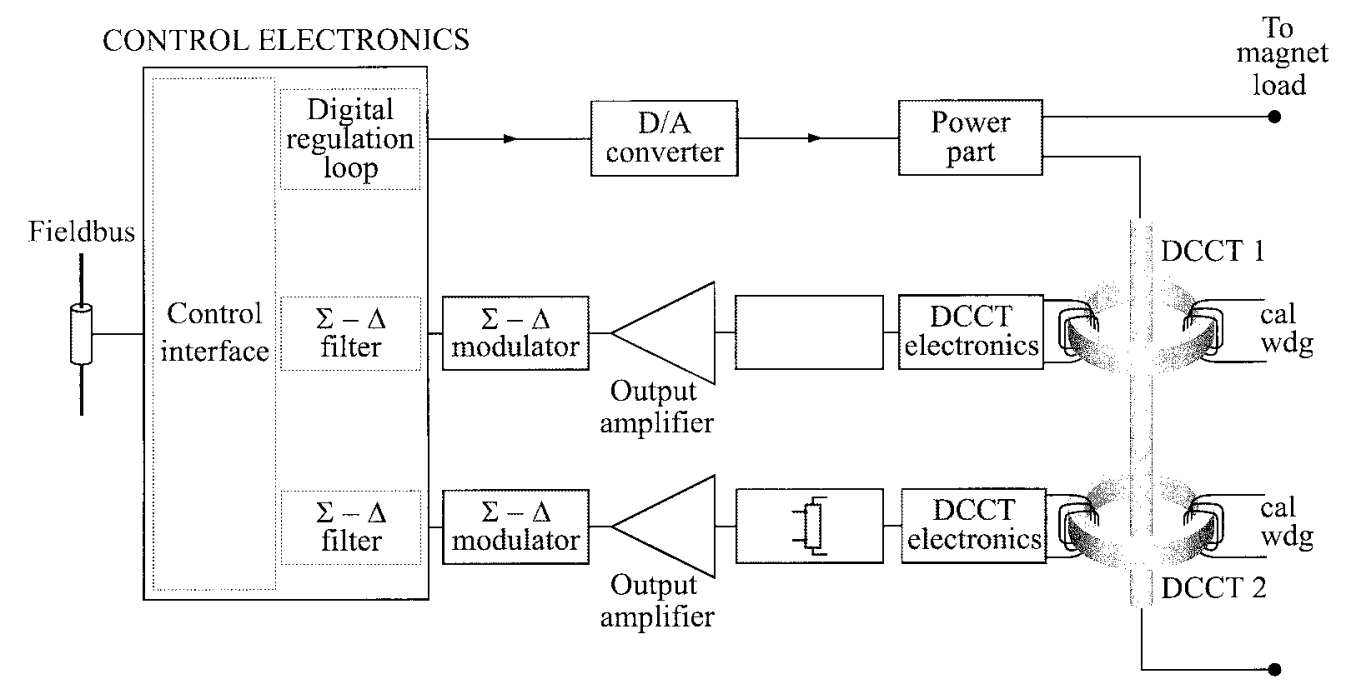

Fig. 3. LHC converter control.

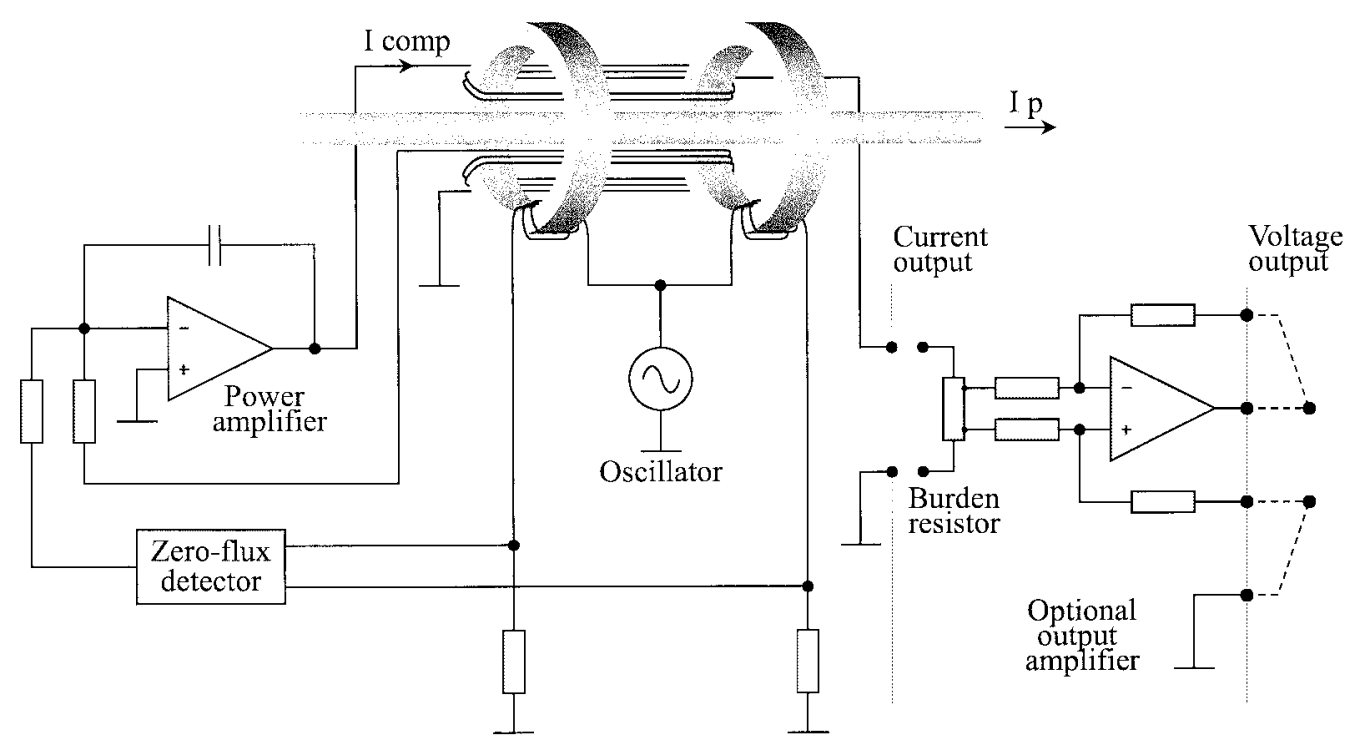

Fig. 4. DCCT principle.

converted to an output voltage in a burden resistor followed by a gain stage and an ADC. In this part it is much more difficult to control the accuracy and, in particular, the long-term drift. Nonlinearity of up to $1 \times 10^{-5} I_{\max }$ has been observed, as well as zero and gain drifts of up to $2 \times 10^{-5} I_{\max }$ in a year.

\section{B. DCCT with Calibration Winding}

The new idea consists of adding a second compensation winding, a "calibration" winding on the toroidal core (see Fig. 5). A known calibration current can then be injected in this winding, producing the same ampere turns as the primary current would, and the normal output of the entire system is verified for accuracy and linearity. Thus, the DCCT/ADC can be fully calibrated in-situ. The current in the main circuit must be zero during the calibration, or at least smaller than the desired uncertainty. More than one DCCT can be calibrated simultaneously. An automated switching system will channel the calibration current to the selected DCCT's.
In the LHC context this will mean eight calibration centers, two in each of the four powering locations, up to $10 \mathrm{~km}$ away from the standards laboratory and $100 \mathrm{~m}$ underground.

\section{The Current Calibrator}

The previously outlined concept requires the ability to produce a known calibration current at the $5 \mathrm{~A}$ level. This current will be generated in a backward connected DCCT, i.e. one that scales the current up instead of down. This new current calibrator (see Fig. 6) is equipped with 12 binary weighted, relay controlled, input windings and an extra oneturn winding fed with a controlled fraction of the current, a technique similar to the current comparator [2]. Several output windings provide different output ranges up to $\pm 10 \mathrm{~A}$. The resolution is limited by the noise and zero stability of the zeroflux detector, which at present is a few microampere-turns. For high-speed operation it would be possible to replace the relays 


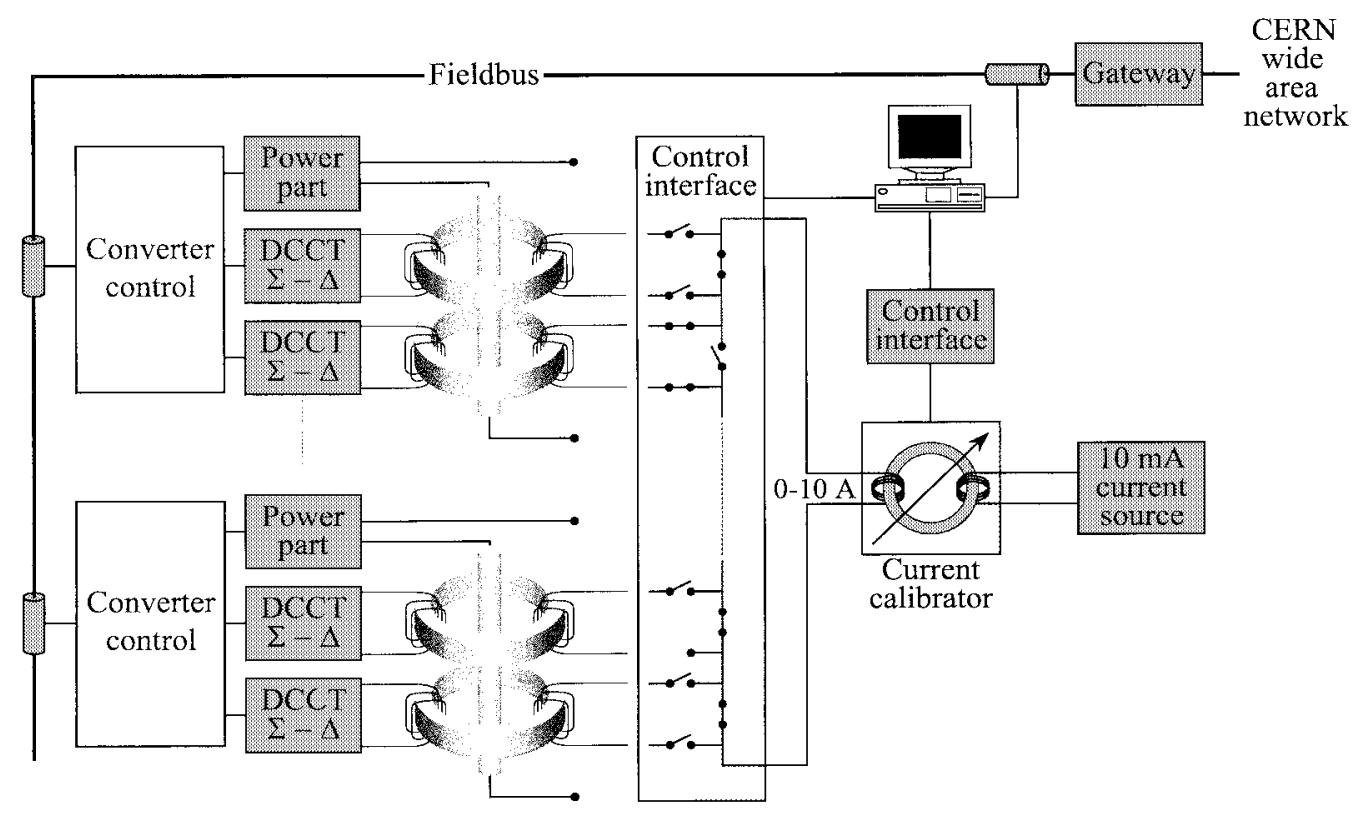

Fig. 5. Calibration system.

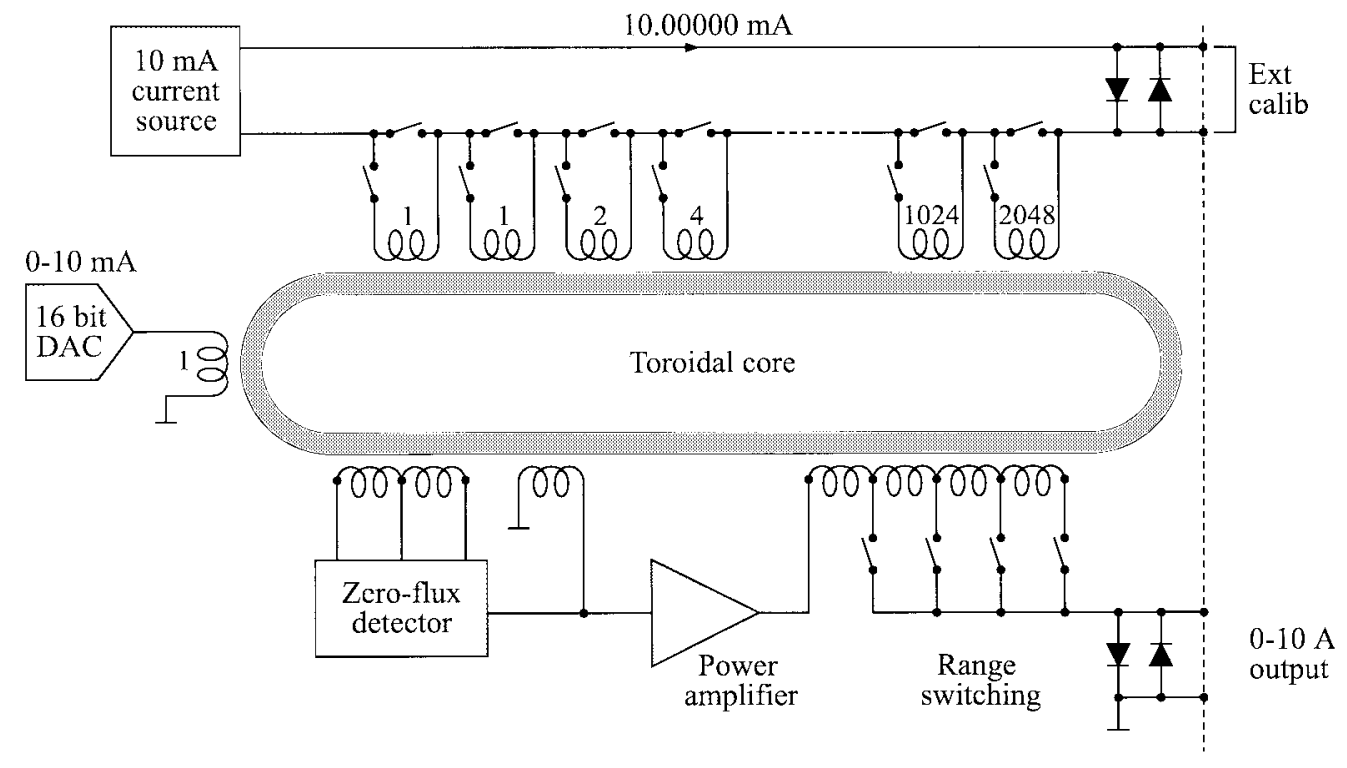

Fig. 6. The current calibrator principle.

with solid-state switches. In the present application the extra development effort does not seem justified.

\section{Current Standard}

The reference input to the calibrator will be provided by a new $10 \mathrm{~mA}$ current standard. An infrastructure will be set up with many such, stationary and portable, $10 \mathrm{~mA}$ standards based in the CERN standards laboratory. Periodic inter-comparison will be performed, much like present systems for primary $10 \mathrm{~V}$ Zener standards. The portable standards will transfer the $10 \mathrm{~mA}$ to the LHC calibration centers around the ring. The inter-comparison procedure will naturally be much less critical than for a voltage standard, as thermal emf's will have a negligible influence.

\section{E. Progress}

DCCT's with calibration windings have been ordered and some have been delivered. Functional tests have been successful. Full accuracy tests will be performed during 1998.

A prototype current calibrator has been working for many months. It has a resolution of more than 24 bits and its zero stability is better than $2 \times 10^{-7} I_{\max }$ over several days. The linearity has been tested against a Guildline 9920 bridge and is of the same order of magnitude. It has already proven to be a versatile and outstanding tool in analyzing the performance of high precision burden resistors.

A series of five $10 \mathrm{~mA}$ current standards have been delivered and they perform as expected. The stability has been better than $3 \times 10^{-7} I_{\max }$ over several weeks. The transfer accuracy 


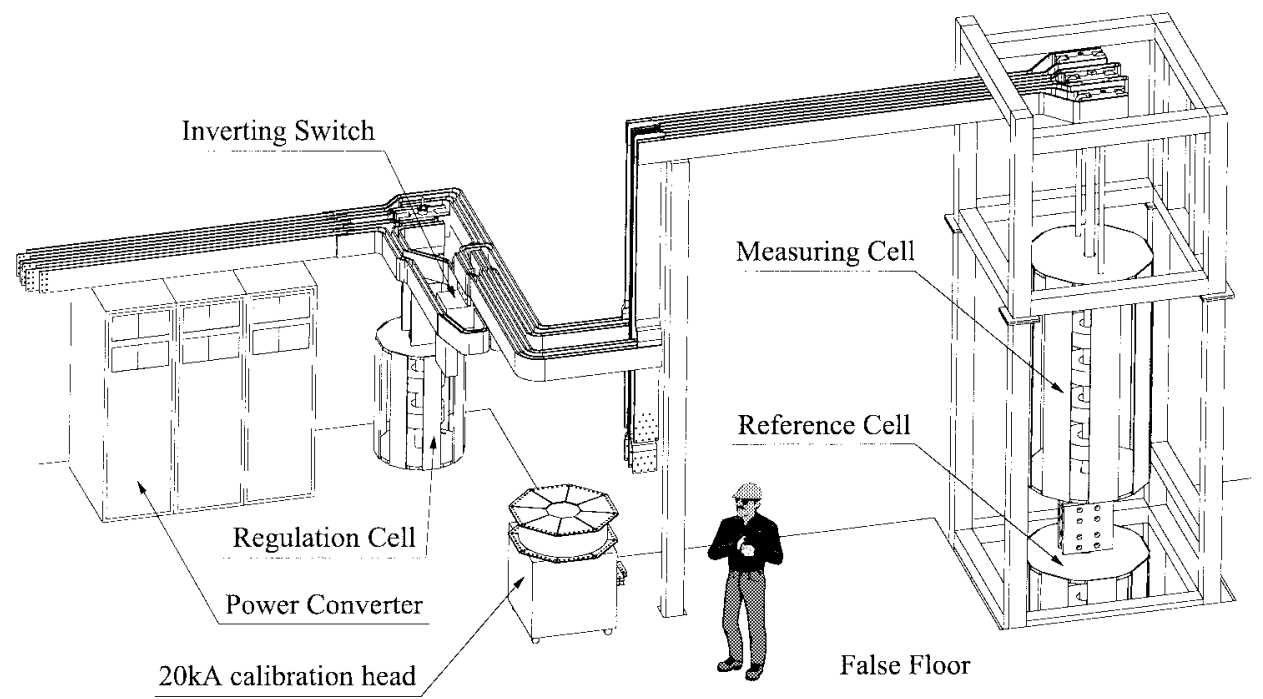

Fig. 7. The $20 \mathrm{kA}$ testbed for DCCT's.

between the units, using the back-to-back method, was found to be around $1 \times 10^{-7} I_{\max }$.

\section{THE $20 \mathrm{kA}$ TESTBED}

A unique facility has been built at CERN that will enable full evaluation of DCCT's up to $20 \mathrm{kA}$. It consists of a power converter, a polarity-changer, a busbar system, and a system of quasicoaxial cages to house the DCCT's for regulation of the power converter, the calibration heads and the DCCT's to be evaluated/calibrated (see Fig. 7).

The power converter is built with soft-commutated, 20 $\mathrm{kHz}$, switch-mode technology to reduce EMC problems to a minimum. It has five modules of $4 \mathrm{kA}$ and $6 \mathrm{~V}$ connected in parallel [3]. The converter is designed with a high-performance regulation system employing feed-forward, to minimize any influence from variations and disturbances on the mains network.

The purpose of the polarity-changer is to facilitate verification of linearity and zero offset problems in the device under test (DUT). It will also serve to produce a complete demagnetization cycle for the magnetic cores in the circuit.

The busbar system is designed with minimum spacing and $\mathrm{a}+/-/+$ configuration to minimize EMC problems, in particular the generation of external fields.

Coaxial cages were designed to create near-ideal working conditions for the DCCT's. One of the cages can be opened with hydraulic $20 \mathrm{kA}$ connectors, to facilitate insertion and removal of the DUT. Facilities are also provided to create field asymmetries to verify the tolerance of the DUT in this respect.

Two calibration heads for $20 \mathrm{kA}$ and $24 \mathrm{kA}$ have been manufactured by industrial partners with a relative uncertainty of less than $1 \times 10^{-6}$. The testbed has been run at $20 \mathrm{kA}$ and commissioning of the calibration heads is well under way [4].

\section{CONCLUSION}

A new concept has been presented to cope with the accuracy requirements of the LHC magnet current control. Prototypes have been built of the key components. Test facilities have been built to prove the validity and the results from these first steps have demonstrated the expected performance. Much work still remains before the complete system is fully proven and can be put in operation.

\section{ACKNOWLEDGMENT}

The author acknowledges the contribution to this work by many more people than can be mentioned here. It is the result of many discussions and reviews with people inside and outside CERN.

\section{REFERENCES}

[1] Available from http://www.cern.ch.

[2] W. J. M. Moore and P. N. Miljanic, The Current Comparator. London, U.K.: Peregrinus, 1988.

[3] F. Bordry, A. Dupaquier, G. Fernqvist, and H. E. Jorgensen, "An LHC 20 kA, 6 V, power converter prototype," 6th Eur. Particle Accel. Conf., EPAC'98, Stockholm, Sweden, 1998.

[4] G. Fernqvist et al., "Design and verification of a $24 \mathrm{kA}$ calibration head for a DCCT test facility," this issue pp. 346-350.

Gunnar Fernqvist, for a photograph and biography, see this issue, p. 350 . 